\title{
The Participation of View in the Perception of Object with the Variative Shape
}

\author{
Losik George $^{1 *}$, Vadim Tkachenko ${ }^{1}$, Igor Boyko ${ }^{1}$ and Alla Bogurina ${ }^{2}$ \\ ${ }^{1}$ United Institute of Informatics Problems of the National Academy of Sciences of Belarus, Europe \\ ${ }^{2}$ Department of Psychology, Belarusian State Pedagogical University, Europe
}

*Corresponding author: Losik George, United Institute of Informatics Problems of the

Received Date: July 22, 2019

National Academy of Sciences of Belarus, Europe.

\section{Abstract}

Aim: to describe that in the perception of the object with a variative shape, the kinesthetic sensations of the joints and the "human hand" system as a whole are involved.

Methods: As objects with the variative shape for perception in all experiments, 12 metal springs were used, different in elasticity, length, diameter of the spiral, pitch of the coil, wire thickness, material, color.

In order to relate the subjective and objective properties of the spring, it was described its color, measured the elasticity of the spring with a dynamometer, the length, the diameter of the spiral, the pitch of the coil and the thickness of the spring wire with a caliper. Eight persons participated in the experiments. participated consequently in three experiments, to evaluate the rate of difference between the same pairs of spring for different combinations of analyzers. For determining the minimum dimension of a psychological space in this space the variativity scale was revealed. This is a complex scale, and therefore it disappears if one or other link of its mechanism is missing.

Results: We assume that there is a superstructure over the self-working visual, tactile, kinesthetic analyzers. This is a superstructure in the form of another, integral signal analyzer from the three analyzers.

The result of this analyzer is the "sensation" of the person of this quality of the object in variativity of its shape. To evaluate this quality, there are no special receptors in humans. Nevertheless, in the phylogeny to evaluate this biologically important quality, a separate scale of evaluation was formed on the behavioral and the cortical levels. An analogue to this analyzer is the object distance from analyzer. Observer for stereopsis vision, by comparison in the visual cortex of two images arriving in the brain from the right and left eyes.

We received matrix scores, which were then processed on a computer. The mechanism of evaluation of elasticity and variativity is realized by hand together with vision. With the help of active influence on the object, a person studies in him "visual" changeability of the shape.

Conclusion: The coordinated work of the visual, tactile and kinesthetic analyzers forms a separate stand-alone analyzer. Due to simultaneous movements of the eyes and hands, this analyzer allows the perceiving person to discover more information for comparing the presented objects of the variative shape and estimating the differences between them. People who are blind from birth cannot, unfortunately, form images of the objects with the variative shape.

Keywords: Perception action; Kinesthetic sensations; Visual analyzer

\section{Introduction}

There are different approaches to the experimental study of a person visual perception of surrounding objects [1-3]. The approaches differ in what kind of a task the researcher is planning to solve: to study the properties of objectivity and integrity of perception [4,5], or to identify work patterns of hands and eyes during perception, or to open the mechanism of perception, or to study the algorithm of forming a mental image of the object [6]. In this paper, we are considering an aspect that was not so important in known studies of perception $[7,8]$. This is the aspect of the object perception when its shape is variative. For the person, the perceived object differs from the another one, first of all, by shape and color [9]. Therefore, to form an image of a new object the person often does not need to touch the object with the hand [7]. The person can perceive the object remotely by sight, recognize by shape and color, or perceive it from TV or computer screen, or a book picture. At the same time, there are a category of objects having the variative shape, for better distinctness of which, the person during perception, wants to sense their weight, flexibility, roughness, amorphousness [7]. To form the image of the object, the object is necessary touch 
with the hand. The attributes of the object like shape, color, texture, roughness, surface temperature can be conditionally called the attributes of the shape. They are easily perceived with the eye and hand and are filling the mental image. Additionally, to the attributes of the object shape, we introduce the attribute "variativity of the shape".

The passive touch with the hand of the object is sufficient to determine the shape, roughness, stickiness, temperature, or with the tongue to determine the taste of the object [10]. It has been called tactile sensations. Besides, there are kinesthetic sensations like sensations in muscles and joints as a result of muscle efforts missing during the passive touch [11].

We focus on perception of the variative object and define it as the physical object, which changes its shape due to the efforts of the hand muscles and visually noticeable. Moreover, the change of the shape does not destroy the physical nature of the object and keeps its functional purpose. For example, the elastic object returns to the initial shape after some influence when the plastic object does not. So, the brittle objects cracked under the influence cannot be considered as the objects with the variative shape.

At the same time, the change in shape that is characteristic of the physical nature of such an object goes on a scale that does not destroy the object and its functional purpose for man. For example, an object that is characterized by elasticity returns after perceptive action on it to its previous form, and an object that is different in plasticity is not returned. For example, objects that differ in "croakiness", acquiring cracks, cannot be considered as objects with a variable form.

The definition of the object with the variative shape is based not on the scientific nature of the object, but on the cognitive nature, or on the nature of the object to become a functional "thing" for the person (according to Emmanuel Kant's terminology), or to stop being the "thing" in the case of its destruction $[12,13]$. The definition is purely psychological, because the object is variative only if the three following conditions, from which the second and third have the subjective and not science nature, are kept. On the first condition, the object must be able to change the shape because of the physical material, for example, to be flexible, plastic, brittle, under the force that the human hand muscles can produce. On the second condition, the person, mustapply the force on the object with the hand, and with the power sufficient for large-scale change of the object shape and clearly visible confirming the fact of the change, i.e. the reflex to the novelty must work. On the third condition, such the influence should not provoke the object to cease to exist and should not provoke the object to lose the anthropological function, which the person plans to use. Noncompliance of all the three conditions means the object is in the category of "object with nonvariative shape".

We believe that the kinesthetic sensations of human joints and the system "human hands" in general are involved in perception of the object with the variative shape. Besides, simultaneously with the hand the visual system works. Therefore, the evaluation of the variativity degree of the object cannot be reduced to the sum of values from two or three analyzers or to the sum of sensations. According to our hypothesis, in ontogenesis the person possesses a separate analyzer, or rather, a cortical part of the analyzer, which does not have its own receptors, but uses receptors of the visual analyzer and kinesthetic receptors of the hand and fingers, to evaluate the object variativity.

\section{Hypothesis}

Theoretically, it is valid to assume two options of a storage mechanism, in a person mental image, of the information about the variativity of the object shape. According to the first option, the quality of variativity are valid to many types of objects, and therefore in phylogeny the person inherited a separate analyzer to evaluate the qualities and a special kind of sensation is functioning. In this case, a person's perceptual evaluation of the qualities of changeability of the object shape can occur only after perception of the unchangeable object and causes following evaluation of the object changeability and study of the variativity of its image. There is a possibility that such evaluation occurs with the hand together with the vision. The hand and vision of the person are detecting as the tactile so the "visual" changeability of the shape [7]. So, according to the second option it can be assumed that there is no such a separate analyzer, and the evaluation of the object variativity is performed by the kinesthetic sensation of the hand performing this evaluation without the participation of the visual analyzer [10]. To test these two alternative hypotheses, we made a polymodal experiment of multiple perception and study of the same objects (12 springs), but in different combinations of two analyzers: visual and kinesthetic.

\section{Materials and Methods}

In all experiments, 12 metal springs were used as objects with the variative shape for perception. They differ in elasticity, length, diameter, pitch, wire thickness, material and color (Figure 1).

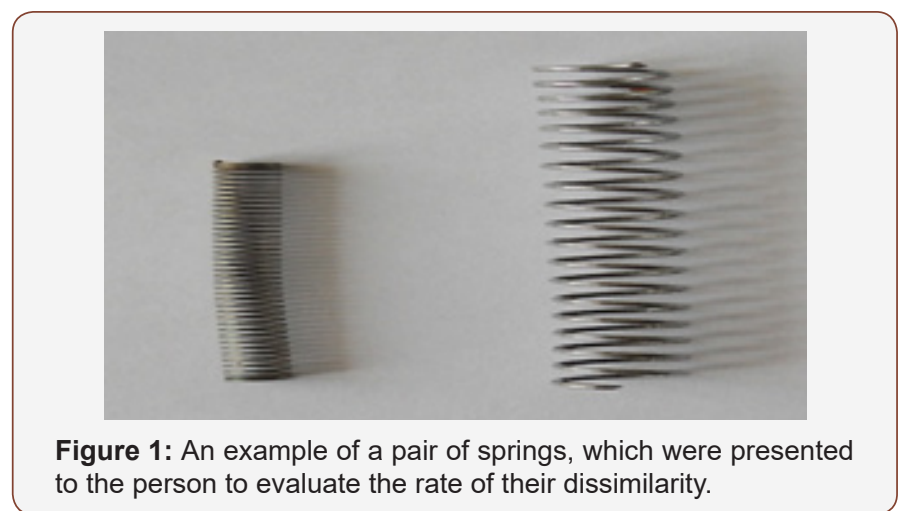

To correlate the subjective and objective properties of the spring,

its color was described, the elasticity coefficient was measured by the dynamometer, the length, the diameter of the spiral, the coil pitch and the thickness of the spring wire were measured with the caliper. These data are presented in the (Table 1). 
Table 1: Objective properties of springs.

\begin{tabular}{|c|c|c|c|c|c|c|c|}
\hline $\begin{array}{c}\text { Spring } \\
\text { Number }\end{array}$ & $\begin{array}{l}\text { Coefficient of } \\
\text { Elasticity }\end{array}$ & $\begin{array}{l}\text { Spring Length } \\
\text { (cm) }\end{array}$ & $\begin{array}{c}\text { Spring Coil } \\
\text { Diameter }(\mathrm{cm})\end{array}$ & $\begin{array}{l}\text { Wire Thickness } \\
\text { (mm) }\end{array}$ & $\begin{array}{l}\text { Coil Density } \\
\text { (col. per cm) }\end{array}$ & $\begin{array}{l}\text { Spring weight } \\
\text { (grams) }\end{array}$ & Spring Color \\
\hline 1 & 3 & 2 & 0.9 & 3 & 0.8 & 10 & Steel \\
\hline 2 & 6 & 4 & 0.9 & 1 & 7,0 & 27 & Gray \\
\hline 3 & 0 & 5 & 0.5 & 9 & 5,0 & 90 & Not \\
\hline 4 & 9 & 9 & 0.6 & 1 & 3,0 & 24 & Copper \\
\hline 5 & 2 & 2 & 0,5 & 2 & 4,0 & 15 & White \\
\hline 6 & 13 & 2,2 & 0,4 & 1,5 & 5,0 & 8 & Not \\
\hline 7 & 4 & 2,4 & 0,4 & 1,3 & 6,2 & 6 & Gray \\
\hline 8 & 11 & 11 & 1,0 & 0,9 & 2,7 & 50 & Gray \\
\hline 9 & 15 & 11 & 1,0 & 0,9 & 5,3 & 45 & The black \\
\hline 10 & 8 & 4 & 0,6 & 1,1 & 8,0 & 35 & Not \\
\hline 11 & 6 & 5 & 0,8 & 0,7 & 2,2 & 45 & Steel \\
\hline 12 & 4 & 6 & 0,9 & 0,9 & 4,0 & 55 & Not \\
\hline
\end{tabular}

The person was not informed about the properties of the springs. Only the experimenter knew about it. It was assumed that potentially the person could choose these properties to evaluate the difference of the springs.

\section{Participants}

Eight persons with the age between 18 and 55, participated in the experiments, three females and five males. All participants were right-handed.

\section{Procedure}

There were three experiments. All 8 persons participated sequentially in all three experiments, in which each of them evaluated the rate of difference between the same pairs of springs with different combinations of analyzers. Before the experiment, for a short time all the springs were shown to each person. The person was allowed to study the springs with one hand only to make it easier for the experimenter to follow up with a video analysis of the tactile-kinesis sensation for marking the rate of spring differences.

\section{The experiment with vision only}

The person evaluated the rate of difference of the pair of springs in visual perception only excluding tactile and kinesthetic analyzers. The person was not able to touch the springs. Based on the visual appearance of the two springs, the person had to rate their difference with a score from 0 (no difference) to 9 (maximum difference), without any hint from the experimenter.

\section{The experiment with the hand only}

The person evaluated the difference between the springs with tactile and kinesthetic perception only excluding the visual analyzer. The person had to rate the difference between the objects only with the right hand based on tactile feeling of the objects. The experiment was recorded on the camera.

\section{The experiment with the eyes and hands together}

Evaluation of the difference rate of the springs was implemented simultaneously by the visual, tactile and kinesthetic perception. The experiment was recorded on the camera as well.
For each experiment, a personal scoring matrix was prepared to be processed by the experimenter on a computer using the multidimensional scaling method (Statistica 8.0 program). In the multidimensional scaling procedure, the first step was to determine the minimum dimension to which the scores of the persons could be extrapolated.

There are several methods for determining the minimum dimension of a psychological space (Torgerson, Shepord, Izmailov $\mathrm{Ch} \mathrm{A}$ ). Izmailov $\mathrm{Ch} \mathrm{A}$ notes that the choice of the dimension for psychological problems is not so important as the choice of a good meaningful interpretation of the scales [14]. For the psychological interpretation of what object qualities are used to find their differences during the test, in our case it was enough to have three scales revealed by computer processing of the scores. The criterion for choosing the dimension was the no increase in the extrapolation accuracy after the dimension "three scales".

The number of scales in the multidimensional scaling in the study of perception is usually two, three or four. The number of scales reflects not the number of physical parameters of the stimulus, but the number of psychological scales as physiological neoplasms in the brain. They compensate a large number of objective parameters of objects by a small number of integral scales. Thus, single-pole objective parameter scales are replaced in the perceptive space with the alternative-pole scales [11].

It was necessary to test the hypothesis of the existence of a complex mechanism or a complex scale of human perceptual evaluation of the variativity of the object shape and to verify that the scale of evaluation of the object variativity using the multidimensional scaling technique is valid and checking that this is a complex scale, which can disappear if one or other link of its mechanism is missing. We assumed that there is a superstructure over the self-working visual, tactile, and kinesthetic analyzers in the form of the integral signal analyzer from the three analyzers. The result of the analyzer work is a person's "feeling" of the quality of the object which is the shape variativity. The person does not have specific receptors to evaluate this quality. Nevertheless, in 
phylogeny, a separate evaluation scale has been formed on the behavioral and cortical levels to evaluate this biologically important quality. The analogue to this analyzer is the analyzer evaluating the distance of the object from the observer on stereo vision comparing in the visual cortex two images coming to the brain from the right and left eyes.

After processing the matrix, the experimenter was able to see on the paper the "location" of 12 points of objects in a threedimensional psychological space. The analysis of the location of the points was made and it was found out what characteristic scales the person chose to distinguish springs. The order and the distance of the object location allowed to interpret what physical property of these objects (springs) is the closest to the scale of the person.

\section{Results}

The results of the single-modal visual evaluation differences of springs (without touching them)

As noted, in the first experiment, the person on a 9-point scale made a decision on the difference of springs, and he could only see them lying on the table in front of the person. The person could view the springs without time limitations, change the direction of the gaze, but the person was not allowed to touch the springs in the course of this experience. Naturally, in this experiment, only the optical properties of the spring, such as its length, color and thickness, could be chosen to distinguish between springs. Characteristic scales revealed by the results of the first experiment in different test persons are presented in (Table 2).

Table 2: Characteristic scales, revealed in the persons in the experiment with a purely visual evaluation of the difference in the springs.

\begin{tabular}{|c|c|c|c|c|c|c|}
\hline \multirow{2}{*}{ The subject } & \multicolumn{6}{|c|}{$\begin{array}{c}\text { The fact of revealing (an indicative scale Di) of a particular physical axis in the respondent's answers using the } \\
\text { multidimensional scaling of the program Statistica }\end{array}$} \\
\hline & Spring Length & Elasticity Springs & Spring Coil Diameter & Spring Wire Thickness & Coil Density & Spring Color \\
\hline Zhenya N & D2 & & D1 & & & \\
\hline Lena P & D1 & & D2 & & & \\
\hline Valya P & D1 & & D3 & & & $\mathrm{D} 2$ \\
\hline Alex Kh & & & & D1 & & \\
\hline Alex S & & & & D1 & & D2 \\
\hline Artiom & & & D2 & D1 & & \\
\hline Katya & D1 & & & & D2 & \\
\hline Victor M & D1 & & D2 & & & \\
\hline Typical scales & $\mathrm{D} 1, \mathrm{D} 2$ & & D1, D2, D3 & D1 & D2 & D2 \\
\hline
\end{tabular}

It can be seen from the table that the test persons in the first experiment did not use such a property as spring elasticity. In this case, visual inspection of the spring most often focused the attention of the persons on its length. Secondly, attention is paid to the diameter of the coil of the spring. The test person constructs the perception of springs on the basis of sensations (measurements), as a rule, of two of any of their qualities, the geometric properties of the shape. This perception, in fact, is close to the "sum of sensations."

- Note: The sign "Di" marked the fact of revealing the scale of the given person in the form of one of the scales of the threedimensional psychological space, and the miss - the fact of the absence of a corresponding scale in the three-dimensional space.
The results of the experiment on unimodal tactilekinesthetic evaluation of the difference in springs (without the participation of vision)

The second experiment with the same person followed a short time after the first. The eyes of the person were tied, and he could only feel with his hand a couple of springs in front of him when he made a decision about their differences. The person touched the spring with his hand, felt it with his fingers, took it into his palm, bent. In this case, it was believed that the person uses tactile and kinesthetic sensations. Identified in different persons by computer processing, the indicative scales based on the results of the second experiment are presented in Table 3.

Table 3: Characteristic scales, revealed in the test persons in the experiment with unimodal tactile-kinesthetic evaluation of the difference in the springs.

\begin{tabular}{|c|c|c|c|c|c|c|}
\hline \multirow{2}{*}{ The subject } & \multicolumn{6}{|c|}{ The fact of detection (the characteristic scale Di) of a specific physical axis in the answers of the subject using the data } \\
processing of the multidimensional scaling program Statistica
\end{tabular}




\begin{tabular}{|c|c|c|c|c|c|c|}
\hline Luda & D1 & & D2 & D3 \\
\hline Typical scales & D1, D2 & D2, D1 & D2 & D3 & D3 & D3 \\
\hline
\end{tabular}

It is natural that in this experiment, when the vision was not used and the differences were estimated by tactile and kinesthetic analyzers, we received from the persons different than in the first experiment, the points of difference of the same springs, other revealed scales correlating with other physical characteristics of springs, weight, density of windings, elasticity.

Based on the analysis of the data in Table 3, it can be concluded that in the second experiment, a spring elasticity scale was used to evaluate the differences between the test persons. Typically, the test persons focused the tactile analyzer's attention on the length of the spring. Second, attention was paid to the assessment of spring elasticity. As in the first experience, we found no unexpected patterns in this experience. As before, the perception of the difference between the springs is usually based on the feelings of their two metric qualities and shape properties. This perception is also close to the "sum of sensations" (Table 3).

- Note: The sign "Di marks the fact of revealing the scale of this person in the form of one of the scales of the threedimensional psychological space, and the missing is the fact that there is no corresponding scale in the three-dimensional space.

\section{The results of the final experiment on the multimodal evaluation of the difference in springs with the participation of vision and tactile sensation}

In the third experiment, the person was finally given the opportunity to evaluate the springs both visually and to the touch. In this experiment, it was checked whether in this case the score is equal to the algebraic sum of the two previous estimates, or whether the hypothesis of new additional information on the elasticity of the shape of the object that the joint work of the hand and eye can reveal is true.
For this, first of all, video shooting of the motor-visual behavior of the persons was analyzed. Videography showed that the person evaluated in points the difference between the two springs presented to him by examining them, feeling both springs with one hand, by applying bending influences on the springs. With the help of a video camera, we recorded the movements of the person's hands and the changes in the position of his head, the direction of his gaze.

On average, the test persons in this experience studied the springs for a little longer before deciding on the significance of their differences. It is important that the test persons did not overshadow the type of bending springs with their hands when they were manipulating them. The person monitored the course of his or her arm's influence on the person with his or her eyes, the changes in the visual pattern, and the transformation of the spring shape under the influence of the hand.

In addition, we, as before, received scores matrices, which were then processed on the computer. Table 4 presents the axes that were identified and interpreted in the third experiment for each person (Table 4).

- $\quad$ Note: The sign "Di" marked the fact of revealing the scale of the given person in the form of one of the scales of the threedimensional psychological space, and the miss - the fact of the absence of a corresponding scale in the three-dimensional space.

Table 4 shows that persons evaluate the difference between springs in this multimodal experiment for a large number of different characteristics. However, the evaluation on the elasticity scale of the spring has become very representative. Evaluation occurs on the visual and tactile signs of the springs in most cases in parallel and together.

Table 4: CCharacteristic axes revealed in subjects in the experiment for a multimodal (visual-tactile) evaluation of springs.

\begin{tabular}{|c|c|c|c|c|c|c|}
\hline \multirow{2}{*}{ The subject } & \multicolumn{6}{|c|}{$\begin{array}{c}\text { The fact of detection (the characteristic scale Di) of a specific physical axis in the answers of the subject using the data } \\
\text { processing of the multidimensional scaling program Statistica }\end{array}$} \\
\hline & Spring Length & Elasticity Springs & Spring Coil Diameter & Spring Wire Thickness & Coil Density & Spring Color \\
\hline Zhenya N & D2 & D1 & & & & \\
\hline Lena P & & D3 & D1 & D2 & & \\
\hline Valya P & D2 & D1 & & & & \\
\hline Alex Kh & & D1 & D2 & & & \\
\hline Alex S & & $\mathrm{D} 2$ & D2 & & & \\
\hline Artiom & D2 & D1 & & & & \\
\hline Katya & D1 & D2 & & & & \\
\hline Victor M & D1 & D3 & & & D2 & \\
\hline Typical scales & D2, D1 & D1, D2, D3 & D1, D2 & D2, D3 & D2 & \\
\hline
\end{tabular}

\section{Discussion}

Based on the study, we can conclude that the first hypothesis is confirmed. Most likely, the evaluation of the elasticity, variativity of the shape of the object occurs after the perception of the statics of a stable object at first. Only after the formation of the static image of the object begins implementation of the mechanism for assessing the variativity of its shape and replenishment of information previously formed image. 
The mechanism of evaluation of elasticity and variativity is realized by hand together with vision. With the help of active influence on the object, a person studies in him "visual" instability of shape. The coordinated work of the visual, tactile and kinesthetic analyzers shapes a separate stand-alone analyzer. Due to simultaneous movements of the eyes and hands, this analyzer allows the perceiving person to discover more information for comparing the presented objects of the variative shape and estimating the differences between them.

Along with this, when assessing the differences between objects from one another (in the absence of prescriptions), such parameters as objects length, weight, color are used. However, the ball score of the pair difference in the objects varies, if the person's hand also makes active influences on the object and transforms its shape.

\section{Conclusion}

1. The definition of the object with the variative shape cannot be based on the scientific nature of the object existence, but on the cognitive nature, on the nature of the object to become an object, a functional "thing" for man. In the definition, the object with the variative shape is a physical object, which changes its shape visually noticeable to the human being due to the efforts of human hand muscles under its perceptual effects. At the same time, the change of the shape peculiar to the physical nature of the object goes on the scale that does not destroy the object and its functional purpose for the human being. Thus, the object, which is characterized by elasticity, returns to its former shape after perceptual influence, and the object, which is characterized by plasticity, does not return. For example, brittle objects, which acquire cracks, cannot be considered as the objects with the variative shape.

2. The category of variative objects may include the object under the conjunctive fulfillment of three conditions, of which the second and third have a subjective, non-scientific nature. According to the first condition, the object must be objectively capable of changing its shape by physical material, for example, it must be flexible, plastic, and brittle due to the influence of force in the range that can be produced by human hand muscles. According to the second condition the person has to make an active force influence on this object with the hand, and with the power sufficient for large-scale objective change of the shape. The change must be of such a scale that the vision can detect the fact of change, i.e. the reflex to novelty must work. According to the third condition, the object must not cease its existence from such active perceptual influence, must not lose the ability to perform the anthropological function, which the person plans to perform.

3. The kinesthetic sensations of the joints and the system of the human hand as a whole are involved in the perception of an object with the variative shape. Along with this, the visual system works simultaneously with the hand. Therefore, the evaluation of the degree of variativity of the object shape from the point of view of the variativity vector cannot be reduced to the sum of the evaluations of two or three analyzers, to the sum of sensations. In order to evaluate the variativity of the object shape in ontogenesis, a separate analyzer is formed, more precisely, the cortical part of the analyzer, which does not have its own receptors, but uses receptors of the visual analyzer and kinesthetic receptors of the hand and fingers.

4. In the process of applying perceptual influences on the object with the variative shape, a person forms an image of the dynamics of the shape of this object in addition to the image of the static shape of the object in the psyche. According to the definition of the object of the variative shape, the blind from birth cannot, unfortunately, have images of objects with the variative shape.

\section{Acknowledgement}

Special thanks to Igor Boyko on aid in editing and translating the article.

\section{Conflicts of Interest}

No conflicts of interest.

\section{References}

1. Hansen VA (1974) Perception of integral objects. In: VA Hansen (Ed.), Leningrad State University, Russia, p.160.

2. Gibson J, Gibson E (1975) Perceptual learning - differentiation or enrichment? Reader for sensation and perception. MSU, Moscow, Russia, pp.181-197.

3. Zinchenko VP (1969) Formation of the visual image. In: VP Zinchenko and NO Vergilez (Eds.), MSU, Moscow, Russia, p.287.

4. Goncharov OA (2011) Topological and metric principles of processing spatial information: perceptual and age patterns. In: OA Goncharov, NE Yemelyanova, Yu N Tyapovkin (Eds.), Psychological journal 32(1): 87-96.

5. Sokolov EN (2003) Perception and conditioned reflex. New view, In: EN Sokolov (Eds.), Moscow State University, Moscow, Russia, p.288.

6. Kremen MA (2000) Proactive adaptation to new living conditions. In: MA Kremen (Ed.), People's Asveta Minsk, Belarus, Europe, pp. 33-36.

7. Losik GV (2008) Human Perceptive Actions: Cybernetic Aspect. Monograph, IUPI. Minsk, Belarus, Europe, p.147.

8. Losik GV (2014) Perceptive actions with objects of invariant form and perception of their elasticity. In: GV Losik and AV Severin (Eds.), $6^{\text {th }}$ Intern Conf on cognitive science: theses of reports, BFU, Kaliningrad, Russia, pp. 402-403.

9. Zinchenko VP (1997) Image and activity. In: VP Zinchenko (Ed.), Moscow State University, Moscow, Russia, p.608.

10. Zaporozhets AV (1967) Perception and action. In: AB Zaporozhets, LA Wenger and VP Zinchenko (Eds.), Nauka, Moscow, Russia, p.326.

11. Losik, GV (2006) Physiological interpretation of psychological scales. In: GV Losik, and VN Shumskaya (Eds.), Cognitive studies, Sat Articles, Minsk, BSPU, Europe, pp. 55-62.

12. Bespalov BP (2005) The concepts of subject and means in the subjectactivity psychology of labor. In the collection: Yearbook of the Russian Psychological Society 2: 73-79.

13. Vartanov AV (2015) The dimension problem in the integration of local analyzers: sensorics and motorics. In: AV Vartanov and GV Losik (Eds.), Modern psychology in the context of transdisciplinary research Materials of the VI interdisciplinary conference, Minsk, BSPU, Issue 6: pp.34-38.

14. Izmailov Ch (1989) Psychophysiology of color vision. In: Ch A Izmailov, EN Sokolov and AM Chernorizov (Eds.), Moscow State University, Moscow, Russia, p.194. 